\title{
Interactive comment on "Numerical modeling of the dynamics of Mer de Glace glacier, French Alps: comparison with past observations and forecasting of near future evolution" by Vincent Peyaud et al.
}

Anonymous Referee \#1

Received and published: 5 May 2020

General comments

Mer de Glace is the largest french glacier, and probably one of the best known to the general public in France. In the context of climate warming, its future becoming is therefore of high societal interest, especially because it is a major tourist attraction of 
In this paper, the authors address the question of the future evolution of the Mer de Glace using a state-of-the-art ice flow model to simulate the 3 next decades considering a large panel of climate scenarios. The model was calibrated using a large set of data (including mass balance measurement, bedrock information, surface ice flow observation among others) and validated over the last decades against historical records. As a key result, a strong retreat of the Mer de Glace - from 2 to $5 \mathrm{~km}$ - must be expected within the next 3 decades.

To my knowledge this is the most advanced model of the Mer de Glace. The new results permit to refine of an earlier prognostic given by Vincent and al. (2014), who used a model of lower complexity. I am very confident it will not only interest the community of glaciologists, but also skateholder of the economic life (e.g. tourism, hydro-power production).

My feeling is that the manuscript is already in a good shape. I don't have any major concerns about the methodology, which is well established. Also the interpretation of results makes sense to me. I think two aspects of the manuscript still have room for moderate improvements (check the details in the main comments below): i) comparison with other studies treating the future of glaciers in the European Alps to "contextualize" your results ii) the presentation of the paper (structuring and English language). I also reported below more specific and technical comments, which I hope will help to improve the paper before it gets published. 


\section{Main comments}

- Section 3 on the ice flow modelling contains a lot of content, which is standard in the Elmer Ice workflow and widely used. I don't see the point of recalling this again (in particular the set of equations) in the body of the paper. I suggest to replace it by appropriate references, or to move it in appendix or supplementary material to keep the paper concise and focused. Only what is specific to the Mer de Glace case should be kept in the paper's body (e.g. the choice of $A$ relative temperate ice, the implementation of given fluxes on the domain borders, ...) Details about the 3D mesh should also be moved to appendix. Last, modelling details normally belong to 'Methods', so I suggest to move a very concise version of Section 3 (Ice flow) to Section 4 (Methods), and possibly give further details in appendix.

- While you mention similar existing Full Stokes-based models, or global glacier models of the Alps in introduction, you make no link at all in the discussion. Typically, questions like "How do your results compared with some recent global modelling by Zekollari and al. or other FS model of individual large-scale glacier such as Aletsch?" or "Is the Mer de Glace expected to resist better than other neighbouring glaciers?" could be addressed in your paper. Comparisons with recent other models, and other glaciers of the Alps would put your results in a broader context. In abstract and conclusion, you write "To our knowledge a comparison to data at this detail is unprecedented": This statement is vague and unsupported in the paper.

- I feel the reading of the paper can be made more efficient if the English was improved by a professional. Line 10-12 p6 is an example of phrasing with repetitions that can be improved.

Printer-friendly version

Discussion paper

Specific comments 
- I do not fully understand your choice of mass balance approach for the forecast simulation. A logical choice would be to simply compute the PDD everywhere instead of doing so at the Tacul gate only, and extrapolating the MB via Eq. (7). PDD can computed everywhere assuming vertical lapse rate for temperature. By doing so, you would release your hypothesis of linearly changing mass balance with respect to $z$. Don't you lose any information by this assumption? You could possibly tune PDDs to fit observation at Tacul in the past.

Eq (7) Something must be wrong with this equation since $b=0$ when $z_{s}=z_{s T A C}$ while it should be $b=b_{T A C}$, isn't it? Also, from $k_{b}=\frac{\partial b}{\partial z}$ one expects $k_{b}$ to be dimensionless. From Eq. 7, one expects instead $k_{b}\left[z_{s}-z_{s T A C}\right.$ to be dimensionless. Please double check. Also you have not defined $z_{s}$ and $z_{s}=z_{s T A C}$.

127-30 p8 'relationship between ice flux at the gate and surface mass balance upstream of the gate': Could you elaborate your method (and possible its limitations) based on the two following questions: i) can your result of 11 years be interpreted as the average time for the ice to travel from the surface to the gate? ii) This '11 year' result is probably highly dependent of the glacier regime (advancing, retreating, highly retreating ...), which is fine if you modelled the retreat over the next 3 decades, but if this is the case, can you elaborate? and thus equivalently justify your sentence 130 : "It is furthermore assumed that this relationship will remain valid in the future."

116 p12 'the modeled glacier is too thick and velocity too high': this surprises me, I would expect that it is too thick because too slow following simple mass conservation principle. How do you explain that ice can be both too thick and too rapid?

- I missed where you describe the 'sensitivity experiments' of Fig. 9 and 10 in your text? I understand that the goal is test the influence of the artificial flux condition at Tacul, which is important as you only model part of the glacier by lack of data.

Printer-friendly version

Discussion paper 
However, this should be explained in the body of the paper, and not being left alone in Fig. 9 and 10.

\section{Technical comments}

Abstract I suggest to start straight the abstract with the 'Mer de Glace' and remove general statements (i.e. the 3 first sentences) to make the abstract more focused.

I 6-7 p1 'Mer de Glace (Mont Blanc area)' $\Rightarrow$ 'Mer de Glace glacier, France'.

Abstract As said earlier, 'To our knowledge a comparison to data at this detail is unprecedented.' is (too?) vague statement and not supported.

Abstract It is hard to evaluate how much represent 2 to $5 \mathrm{~km}$ without having any prior idea of the glacier size, could you instead use (or add) a relative metric? i.e. $30 \%$ to $65 \%$ of the glacier length.

I 21 p1 Suggest changing 'fluctuations' into 'evolutions'.

I 4 p2 'Process-based model were also developed to take into account simple dynamics (...)' gives the feeling that first physically-based ice flow models started in 2015, which is obviously not the case. Could you reference early SIA-based models applied to mountain glaciers to fix this?

I 8-14 p2 You should clearly write here that by 3D physical model, you mean Stokes without any shallow-like approximations that would reduce the dimension of the equations (unless I'm mistaken?).

10-14 p2 Can you reduce the number of citations? Do you need all of them? It is anyway not exhaustive. 
I 19-21 p2 'In addition, running simulations ... over three decades.' This is crucial here to justify the use of 3D Full Stokes to capture the complexity of the Mer de Glace ice flow, however, this is not exactly what this sentence is doing. I suggest to reformulate it to emphasize that using such a state-of-the-art model is not just an additional opportunity, but necessary to model the complex ice flow. Otherwise the reader might wonder why a simple model can not do the job.

I 21 p2 'This dynamics make it necessary to take into consideration the delay in the glacier response to climatic forcing.' This justifies to take into account the ice dynamics, but not to use a sophisticated model. Just make sure the argument is used for the right reason.

1 31-32 As shown in Fig. 1, Mer de Glace glacier has a single tributary glacier, which is named Leschaux glacier.

15 p3 'maintained' $\Rightarrow$ 'that are monitored'

16 p3 I expected to have the elevations given.

$18 \mathrm{p} 3 \ldots$ they were not ...

111 p3 'paucity' $\Rightarrow$ 'lack'

113 p3 .. the model domain was restricted

114-15 p3 A transition causality word to connect the two last sentences is missing.

119-20 p3 Again can you reduce the number of citations?

129 p3 Rheological parameter with a constant value assuming temperate ice $(A=5.0159$ $e^{-24}$ ), do you mean $10^{-24}$ ? Could you give a reference for this value? Possibly consider change the unit into $158 \mathrm{MPa}^{-3} \mathrm{a}^{-1}$. This is twice the value of Cuffey and Paterson (2010). 
I 8-9 p6 The two next sections describe in detail the respective boundary conditions for the two steps (hindcast and forecast) defined at the surface (mass balance) and at the Tacul and Leschaux gates (ice flux from the accumulation areas), respectively.

I10-12 p6 4 times 'surface mass balance' in 3 lines, can you improve the text? (or use an acronym)

I 8 p7 Define RCP and shortly comment of the meaning of the 3 pathways.

110 p7 'good performance', this statement calls for ad hoc references.

$117 \mathrm{p} 7$ 'is underestimated by climate models': Do you mean in general or for the climate model you specifically use?

121-22 p 7 For each scenario, the correction factor for precipitation is evaluated over the past period 1979-2015.

I 38 p10 'Fig.4.' $\Rightarrow$ 'Fig. 4.'

16 p8 'using the diagnostic formulation for SIA' $\Rightarrow$ 'using the SIA'. Why qualifying the SIA formula as diagnostic?

) and (10) Implementing Eq (10) straight without any correction sounds hazardous as the chance to get unrealistic velocities is high as the SIA model is local (sensitivity w.r.t $\nabla s$ ) and not tuned. In contrast, adding a correction factor that multiply Eq. (10) and is tuned to ensure continuity in time between Eq. (9) and (10) would make sense.

Eq. (10) As you know the thickness here, did you consider implementing the SIA with a "shape factor" that accounts for lateral resistance of the ice flow as function of the type of profile? 
114 p8 'his maximum central value of the velocity', do you mean 'maximum value along the transect'?

115 p8 'above transverse velocity profile': refer to Eq. (9).

116 p8 'Despite the differences in the methods' I'm not sure what are the 2 methods you referring: Eq. (9) vs Eq. (10) ? Please, clarify.

128 p8 Repeat the reference to Durand for the Safran reanalysis.

115 p11 It can be surprising to see so tiny differences between different RCPs. You may say that this was expected as RCPs differ substantially more after 2050 (if l'm not mistaken).

I 30 p11 "However, these strong differences in ice thickness and ice flux at the Tacul gate lead to much smaller absolute differences in thinning and ice flow velocity downstream of the gate; the lower the cross section, the smaller the response differences for the different scenarios." If you were normalizing the model discrepancies by the ice thickness or ice velocity at the gate, the differences would probably be more uniform, isn't it?

11 p12 'assess' $\Rightarrow$ 'estimate' or 'forecast' or 'predict'

$15 \mathrm{p} 13$ 'which is low relative to the mean ablation measured on the tongue of Mer de Glace' can you give a number?

16 p13 'Other uncertainties arise from the linear extrapolation of ablation over the tongue (Eq. (7)) based on measurements in an area of clean ice', your assumption of linear MB w.r.t altitude might by the highest source of uncertainty? If yes, it would make sense to start with this.

18 p13 Please reference to a Figure. 
110 p 13 'is also well known' $\Rightarrow$ 'is also well constrained'

I11 p 13 not underestimated by the model ... But Fig. 5 shows a slight but real systematic over-estimation of velocities, isn't?

I12 p 13 To what 'discrepancies' do you refer? In the sentence before, you say there is no major discrepancies in velocities.

11-4 p14 One can also argue an inaccurate sliding ratio to explain model errors? Have you considered testing different rate factors $A$ to increase or decrease vertical deformation?

113 p15 'will start to increase ...' $\Rightarrow$ 'is expected to increase ..., check not to use future tense when you describe model results, but conditional or equivalent. Same for the sentence right after.

Fig 2. smb not defined. How is it possible that different RCPs show different values in the past times?

Fig 4. formulation(Eq. (10)),

Fig 4. observed surface velocity, ice thickness, and a velocity distribution given

Fig. 6 "Same as Fig. 5, but for surface elevation." Please make a full sentences. For convenience, better to repeat the text to make each individual caption selfcontained. An other possibility is to merge Fig. 5 and Fig. 6, with two panels. The same for Figs. 9 and 10.

Fig. 8 colorbar is missing

Fig. 10 Color coding missing. Again the Figure would be better if self-contained without having to look for the color coding in another Figure. Otherwise merge Fig. 9 and 10 with two panels. 
Interactive comment on The Cryosphere Discuss., https://doi.org/10.5194/tc-2020-75, 2020.

Interactive

comment 